\title{
In Vitro Evaluation of Different Approaches and Dissolution Enhancement Technique of Poorly Water-Soluble Drug Ibuprofen
}

\author{
A.S.M. Monjur-Al-Hossain ${ }^{{ }^{*}}$, Konika Rani Dutta ${ }^{2}$ and Md. Lokman Hossain ${ }^{2}$ \\ ${ }^{1}$ Department of Pharmaceutical Technology, Dhaka University, Dhaka-1000, Bangladesh \\ ${ }^{2}$ Pharmacy Discipline, Khulna University, Khulna-9208, Bangladesh
}

(Received: 9 June 2016; Accepted: 21 June 2016)

\begin{abstract}
Ibuprofen Solid Dispersion (SD) was prepared by simple Physical Mixing (PM) and Kneading Method (KM). In these two cases, four polymers named poloxamer 407, sodium carboxy methyl cellulose, croscarmellose sodium and pregelatinized starch were used to enhance the dissolution profile of Ibuprofen. In both methods, the ratio of drug and carrier were 1:1, 1:2, 1:3 of which the ratio of 1:3 in KM gave comparatively better result than PM method. In vitro dissolution study was performed in distilled water in $50 \mathrm{rpm}$ and at $37 \pm 0.5^{\circ} \mathrm{C}$. In case of pure Ibuprofen, dissolution rate was only $26 \%$ after 60 minutes (mins) of dissolution. While in KM, Ibuprofen pregelatinized starch formulation at 1:3 ratio showed better dissolution rate. After 60 mins, dissolution rate of Ibuprofen was $72 \%$. The SD formulations of Ibuprofen-pregelatinized starch and Ibuprofen-Na-CMC of physical mixing and kneading techniques (1:3 ratio) were characterized by Fourier Transform Infrared Spectroscopy (FTIR).
\end{abstract}

Keywords: Ibuprofen, Solid dispersions, Physical mixing, Kneading method, Dissolution, FTIR

\section{Introduction}

Prospective drug candidates are characterized by a poor oral bioavailability. Often poor drug dissolution/solubility rather than inadequate penetration through the epithelia of the gastrointestinal tract are responsible for poor oral bioavailability ${ }^{1}$. Thus, a key property is aqueous solubility of any therapeutically active substance which governs dissolution, absorption and thus the in vivo efficacy ${ }^{2}$. Drugs with low aqueous solubility have low dissolution rates and therefore, suffer from oral bioavailability problems ${ }^{3}$.

When a drug is administered orally, it firstly dissolves in gastric and or intestinal fluids and then permeates the membranes of the GI tract to reach systemic circulation. Therefore, a drug with low aqueous solubility will typically exhibit dissolution rate limited absorption, and a drug with poor membrane permeability will typically exhibit permeation rate limited absorption.

Therefore, two areas of pharmaceutical research that highlight improving the oral bioavailability of active agents include: enhancing solubility and dissolution rate of poorly water-soluble drugs and enhancing permeability of poorly permeable drugs ${ }^{4}$.

Lots of the research that has been notified on solid dispersion technologies involves drugs that are poorly water-soluble and highly permeable to biological membranes as with these drugs, dissolution is the rate limiting step to absorption. Hence, the assumption has been that the rate of absorption in vivo will be concurrently accelerated with an increase in the rate of drug dissolution. In the Biopharmaceutical Classification System (BCS), drugs with low aqueous solubility and high membrane permeability are categorized as Class II drugs 5 . Therefore, solid dispersion technologies are particularly promising for improving the oral absorption and bioavailability of BCS Class II drugs.

A variety of procedures like particle size reduction, micronization, physical modifications, nano-suspension, modification of crystal habit such as, polymorphs, pseudo polymorphs, complexation, solubilization, salt formation, and use of cyclodextrins can improve the solubility \& dissolution rate of insoluble drug, but these techniques having some practical restrictions, solid dispersion technique overcome this practical limitations. However, the major challenge with the design of oral dosage forms lies with their poor bioavailability. Solid dispersion technique can be used to improve the solubility, dissolution rate and absorption of several insoluble drugs ${ }^{6}$.

Ibuprofen is a nonsteroidal anti-inflammatory drug (NSAID) derivative of propionic acid used for relieving pain, helping with fever and reducing inflammation ${ }^{7,}$. Ibuprofen is a non-selective inhibitor of cyclooxygenase, an enzyme involved in prostaglandin synthesis via the arachidonic acid pathway. Ibuprofen might be considered a weaker antiinflammatory than other NSAIDs ${ }^{9}$. It is practically insoluble in water but very soluble in most organic solvents (like ethanol, methanol, acetone and dichloromethane $)^{10}$. The chemical name of Ibuprofen is (RS)-2-(4-(2-methylpropyl) phenyl) propanoic acid. Chemical structure is<smiles>CC(C)Cc1ccc(C(C)C(=O)O)cc1</smiles>

According to BCS Classification system, Ibuprofen belongs to class II group which is poorly soluble, but highly permeable. To ensure its maximum bioavailability, it has been the recent endeavor to enhance the dissolution property to meet its ultimate bioavailability. Solid dispersion technique can be used to improve dissolution of poorly water-soluble drugs such as Ibuprofen ${ }^{11,12}$.

\section{Materials and Methods}

\section{Materials}

Ibuprofen, Poloxamer 407, Sodium carboxy methyl cellulose (Na-CMC), Croscarmellose sodium, Pregelatinized starch were obtained as a generous gift sample from Beximco Pharmaceuticals Ltd, Bangladesh. Methanol 
$(\mathrm{M}=32.04 \mathrm{~g} / \mathrm{mol})$ and Absolute Ethanol $(\mathrm{M}=46.07 \mathrm{~g} / \mathrm{mol})$ were obtained from Merck, Germany.

\section{Methods}

In this present study, we concentrated over two methods of SD. These are Physical Mixture (PM) and Kneading Method (KM).

\section{Preparation of Binary PM of Ibuprofen-Polymer}

Physical mixtures of Ibuprofen with poloxamer 407, sodium carboxy methyl cellulose, croscarmellose sodium, pregelatinized starch were prepared by mixing in a mortar and pestle for 15 minutes. Then these mixtures were stored in desiccators at a room temperature until further use.

\section{Preparation of Binary KM of Ibuprofen-Polymer}

The weighted quantities of Ibuprofen with pregelatinized starch, Na-CMC, poloxamer 407 and croscarmellose sodium were triturated in a glass mortar with a small volume of methanol. Kneading of thick slurry was done for 45 mins and then dried at $50{ }^{0} \mathrm{C}$ to constant weight. The dried mass was pulverized properly ${ }^{13}$.

Formulations by these two methods were prepared in three proportions such as 1:1, 1:2 and 1:3. Each formulation was done in triplet.

\section{In-vitro Dissolution Studies of Ibuprofen}

Dissolution studies of pure Ibuprofen as well as the SDs samples prepared by PM and KM were performed using the dissolution apparatus II (USP) with the paddle rotating at $50 \mathrm{rpm}$ in $900 \mathrm{ml}$ of distilled water as dissolution medium at $37 \pm 0.5{ }^{\circ} \mathrm{C}$.

$5 \mathrm{ml}$ of samples was withdrawn at 10 mins intervals started from 10 mins up to 60 mins periodically and replaced with fresh dissolution medium and Absorbance values of the solution were determined with the help of UVspectrophotometer (UV-mini-1240, SHIMADZU CORP., Kyoto, Japan) at $\lambda_{\max } 222 \mathrm{~nm}$, using distilled water as blank against the calibration curve. Dissolution studies were then performed.

\section{Infrared Spectroscopy}

Infrared spectroscopy was performed in IR, Shimadzu, Japan by scanning the sample in potassium bromide $(\mathrm{KBr})$ discs. From dissolution studies, some formulations prepared by $\mathrm{PM}$ and $\mathrm{KM}$ were selected according to comparative better dissolution result and these complexes were analyzed by FTIR and the intermolecular interaction of complex

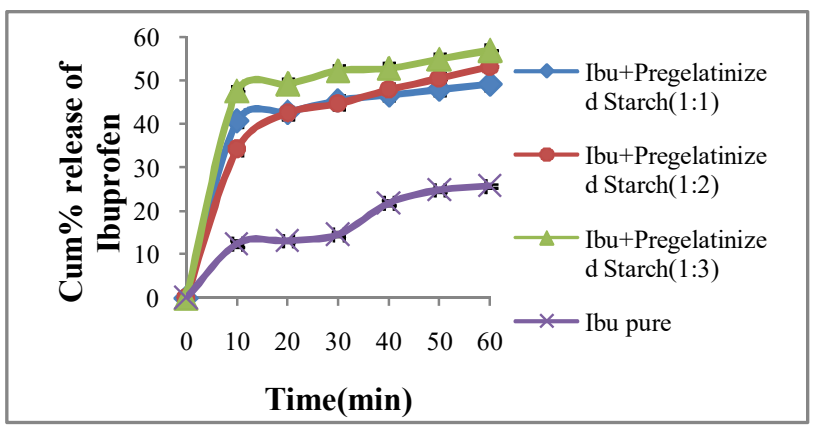

(A) system was established by FT-IR. The sample of pure drug, pure polymers and the solid dispersions containing both the drug and polymer were scanned over the frequency range $4000 \mathrm{~cm}^{-1}$ to $400 \mathrm{~cm}^{-1}$. The IR spectra of solid dispersions were compared with standard IR spectra of pure Ibuprofen.

\section{Results and Discussion}

In-vitro release studies

In this experiment solid dispersion of Ibuprofen with pregelatinized starch, Na-CMC, poloxamer 407 and croscarmellose sodium were performed in two methods. Among these polymers, Ibuprofen with pregelatinized starch and Na-CMC showed comparatively better result than poloxamer 407 and croscarmellose sodium at all ratios. Comparatively better dissolution profile was observed at 1:3 ratios. In vitro dissolution studies were conducted on three times of each of the formulations. The cumulative release of Ibuprofen in dissolution media was only $26 \%$ after 60 minutes which is shown in Figure 1.

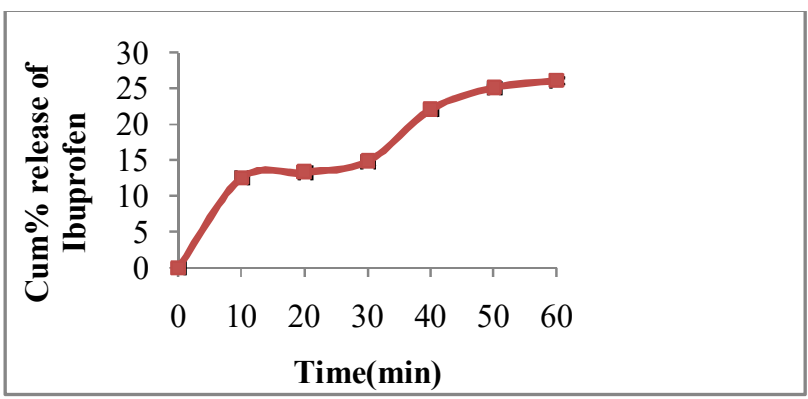

Fig. 1. Cumulative percent release of $50 \mathrm{mg}$ pure Ibuprofen

Figure 2 and 3 shows the zero order and Higuchi release curves of Ibuprofen in PMs technique. As maximum as $48 \%$ Ibuprofen was found to be released in case of physical mixtures (PMs) with pregelatinized starch (1:3 ratio) after first 10 minutes of dissolution. It was $45 \%$ for $\mathrm{Na}-\mathrm{CMC}$ and only $13 \%$ for pure Ibuprofen. After 60 minutes, cumulative percent release of Ibuprofen was $58 \%$ for pregelatinized starch and $52 \%$ for $\mathrm{Na}-\mathrm{CMC}$, but for pure Ibuprofen it was only $26 \%$. In addition, for poloxamer 407 and croscarmellose sodium, it was $50 \%$ and $49 \%$, respectively.

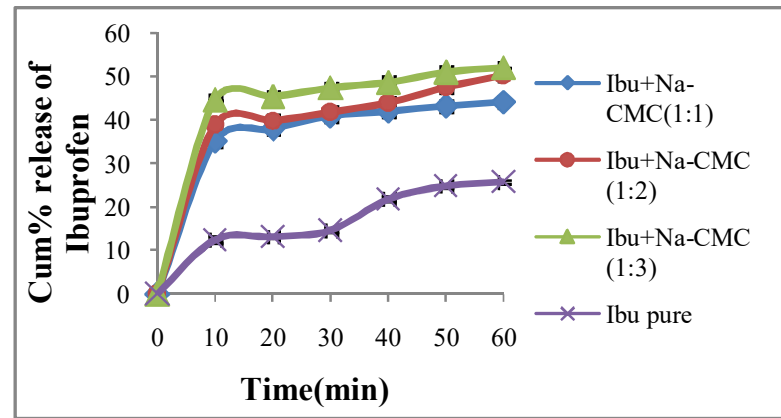

(B)

Fig. 2. Zero order release curves of Ibuprofen from physical mixtures of Ibuprofen with pregelatinized starch (A) and Na-CMC (B) $(n=3)$ 


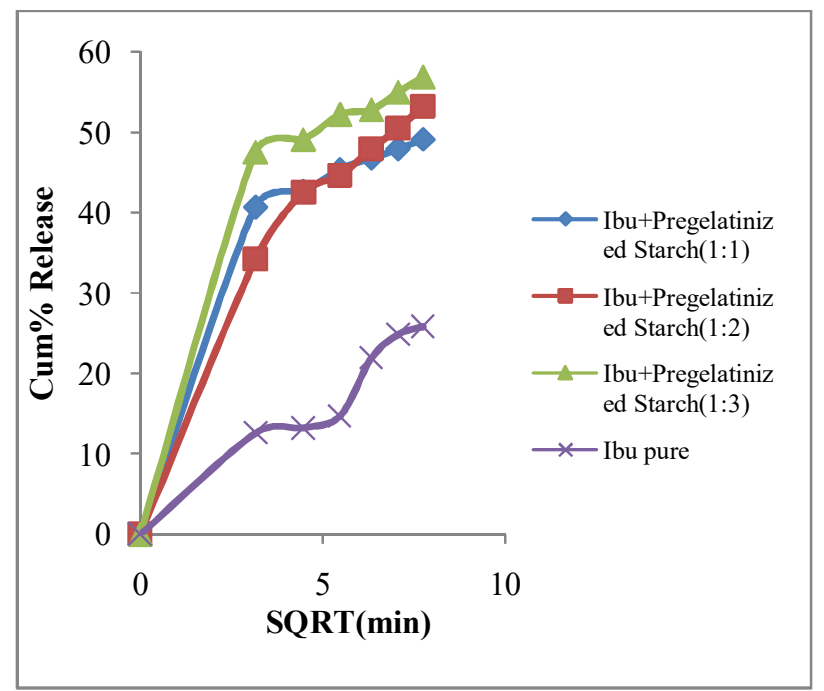

(A)

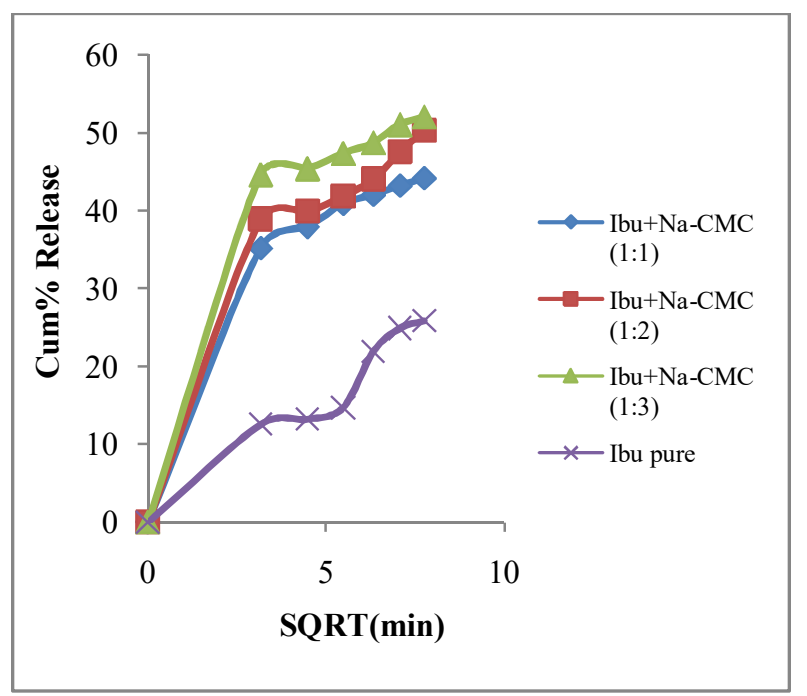

(B)

Fig. 3. Higuchi release curves of Ibuprofen from physical mixtures of Ibuprofen with pregelatinized starch (A) and Na-CMC (B) (n=3)

Figure 4 and 5 shows the zero order and Higuchi release curves of Ibuprofen in kneading method. 60\% Ibuprofen was found to be released in case of $\mathrm{KMs}$ with pre gelatinized starch (1:3 ratio) after first 10 minutes of dissolution and it was $48 \%$ for Na-CMC. After 60 minutes, cumulative percent release of Ibuprofen was $72 \%$ for pregelatinized starch and $66 \%$ for Na-CMC. Furthermore, for poloxamer 407 and croscarmellose sodium, it was $62 \%$ and $60 \%$ respectively.

Figure 6 shows that cumulative \% release of Ibuprofen is better in KMs than PMs.

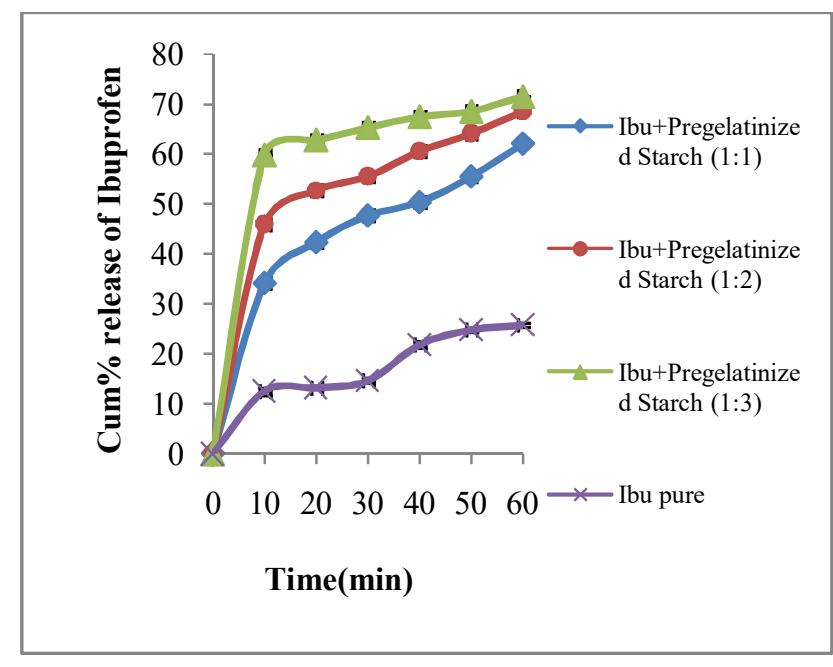

(A)

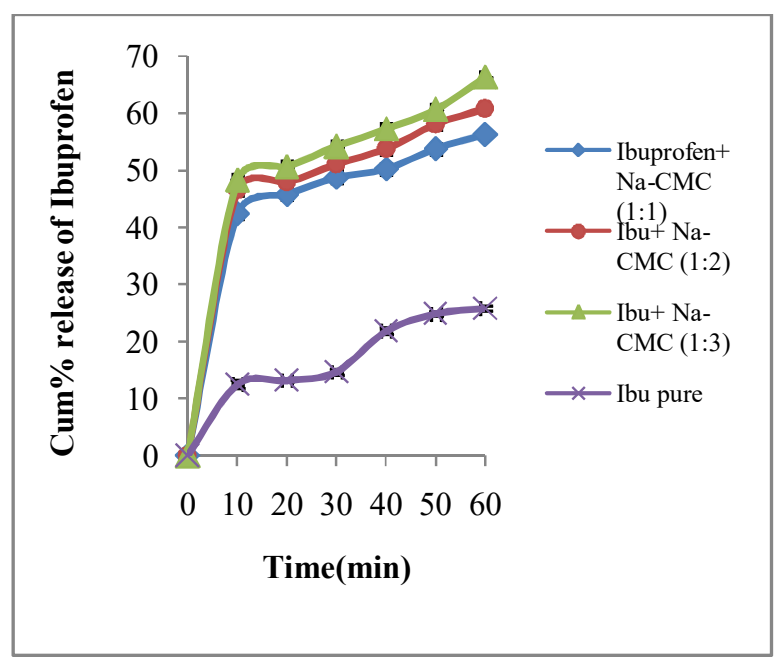

(B)

Fig. 4. Zero order release curves of Ibuprofen from Kneading Methods of Ibuprofen with pregelatinized starch (A) and Na-CMC (B) $(\mathrm{n}=3)$ 


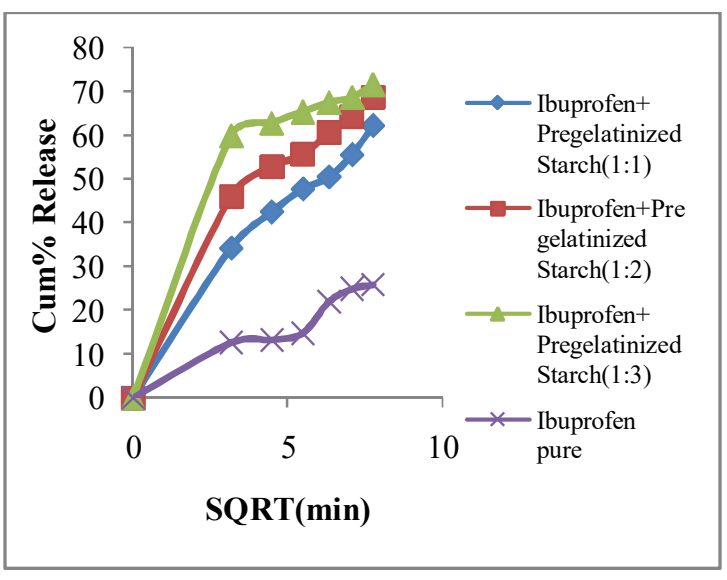

(A)

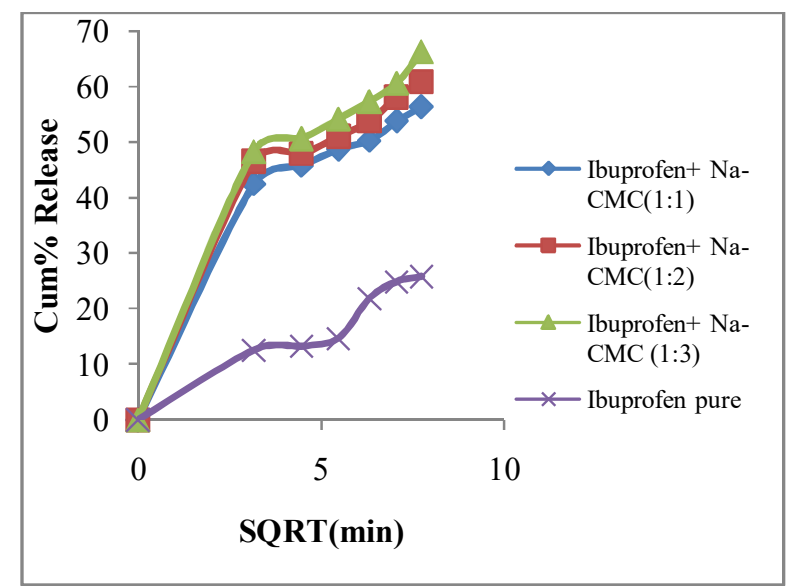

(B)

Fig. 5. Higuchi release curves of Ibuprofen from Kneading Methods of Ibuprofen with pregelatinized starch (A) and $\mathrm{Na}-\mathrm{CMC}(\mathrm{B})(\mathrm{n}=3)$

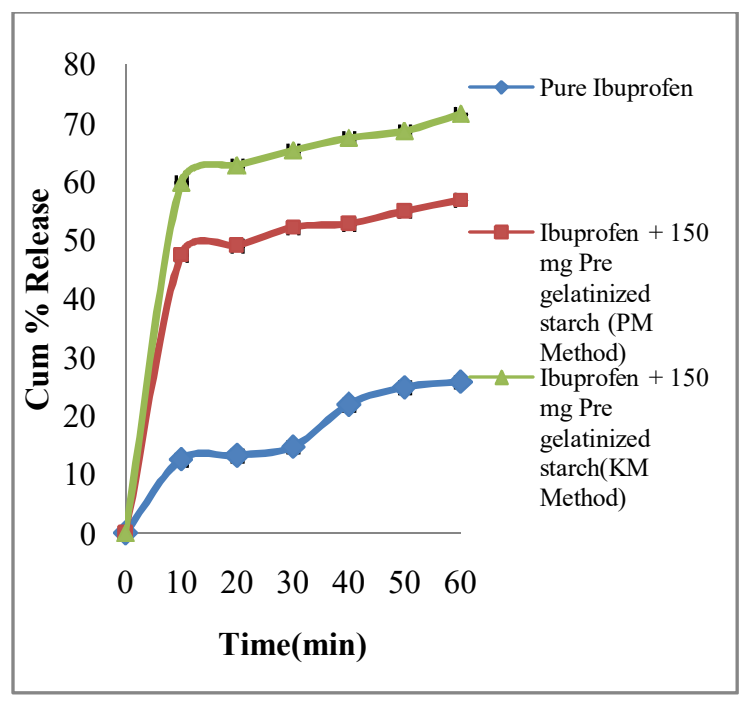

(A)

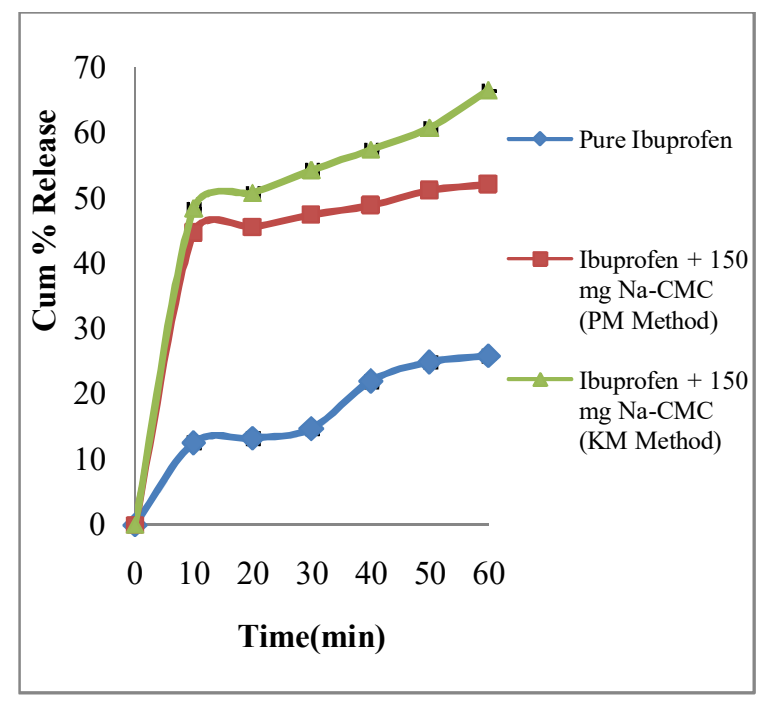

(B)

Fig. 6. Comparison of zero order release curves of Ibuprofen from Kneading Methods of Ibuprofen with pregelatinized starch (A) and Na$\mathrm{CMC}(\mathrm{B})(\mathrm{n}=3)$

The release curve of the drug for both PM and KM showed poor fit for zero order kinetics (Table 1 and Table 2). On the other hand, the delivery system showed better linearity for Higuchi release kinetics. It indicates that drug release was a diffusion process. The dissolution data was best fitted to the Korsmeyer equation, which is often used to describe the drug release behavior from polymeric systems.

$$
\log \left(\mathrm{M}_{\mathrm{t}} / \mathrm{M}_{\mathrm{f}}\right)=\log \mathrm{K}+\mathrm{n} \log \mathrm{t}
$$

Where $M_{t}$ is the amount of drug release at time $t ; M_{f}$ is the amount of drug release after infinite time; $\mathrm{k}$ is a release rate constant incorporating structural and geometric characteristics of the dosage form; $\mathrm{n}$ is the diffusion exponent indicative of the mechanism of drug release $\mathrm{e}^{14}$. As values of diffusion exponent for all the batches were below the standard, it indicated about the fickian diffusion controlled release of Ibuprofen ${ }^{15}$.

However, the goodness of fit for various models investigated for the PMs and KMs of Ibuprofen and polymer was found as following order: Peppas Korsmeyer $>$ Higuchi Model $>$ First Order $>$ Zero Order e.g. the best fitted model was Peppas Korsmeyer's model. 
Table 1. Kinetic parameters of Ibuprofen release curves (physical mixture)

\begin{tabular}{|c|c|c|c|c|c|c|c|c|c|}
\hline \multirow{2}{*}{ PM } & \multirow{2}{*}{ Ratio } & \multicolumn{2}{|c|}{ Zero Order } & \multicolumn{2}{|c|}{ First Order } & \multicolumn{2}{|c|}{ Higuchi Model } & \multicolumn{2}{|c|}{$\begin{array}{l}\text { Peppas } \\
\text { Korsmeyer }\end{array}$} \\
\hline & & $r^{2}$ & $\mathrm{~K}_{0}$ & $r^{2}$ & $\mathrm{~K}_{1}$ & $r^{2}$ & $\mathrm{~K}_{\mathrm{h}}$ & $\mathrm{r}^{2}$ & $\mathrm{n}$ \\
\hline \multirow{3}{*}{ IBP: PS } & $1: 1$ & 0.54 & 0.59 & 0.52 & 0.003 & 0.81 & 5.88 & 0.98 & 0.11 \\
\hline & $1: 2$ & 0.69 & 0.71 & 0.79 & 0.004 & 0.92 & 6.59 & 0.99 & 0.25 \\
\hline & $1: 3$ & 0.53 & 0.68 & 0.61 & 0.004 & 0.79 & 6.73 & 0.95 & 0.09 \\
\hline \multirow{3}{*}{ IBP: Na CMC } & $1: 1$ & 0.57 & 0.55 & 0.62 & 0.003 & 0.83 & 5.4 & 0.99 & 0.13 \\
\hline & $1: 2$ & 0.61 & 0.61 & 0.65 & 0.003 & 0.85 & 5.9 & 0.85 & 0.14 \\
\hline & $1: 3$ & 0.52 & 0.62 & 0.58 & 0.003 & 0.79 & 6.15 & 0.90 & 0.09 \\
\hline \multirow{3}{*}{ IBP: CCS } & $1: 1$ & 0.73 & 0.57 & 0.79 & 0.003 & 0.93 & 5.19 & 0.98 & 0.24 \\
\hline & $1: 2$ & 0.66 & 0.62 & 0.74 & 0.003 & 0.89 & 5.83 & 0.98 & 0.19 \\
\hline & $1: 3$ & 0.51 & 0.58 & 0.55 & 0.003 & 0.78 & 5.82 & 0.99 & 0.09 \\
\hline \multirow{3}{*}{ IBP: POL407 } & $1: 1$ & 0.76 & 0.61 & 0.83 & 0.003 & 0.95 & 5.52 & 0.97 & 0.27 \\
\hline & $1: 2$ & 0.66 & 0.62 & 0.74 & 0.003 & 0.89 & 5.89 & 0.99 & 0.19 \\
\hline & $1: 3$ & 0.50 & 0.58 & 0.55 & 0.003 & 0.77 & 5.85 & 0.94 & 0.08 \\
\hline Pure IBP & - & 0.90 & 0.39 & 0.92 & 0.002 & 0.96 & 3.31 & 0.83 & 0.45 \\
\hline
\end{tabular}

Table 2. Kinetic parameters of Ibuprofen release curves (Kneading Method)

\begin{tabular}{|c|c|c|c|c|c|c|c|c|c|}
\hline \multirow[t]{2}{*}{$\mathrm{KM}$} & \multirow{2}{*}{ Ratio } & \multicolumn{2}{|c|}{ Zero Order } & \multicolumn{2}{|c|}{ First Order } & \multicolumn{2}{|c|}{ Higuchi Model } & \multicolumn{2}{|c|}{$\begin{array}{l}\text { Peppas } \\
\text { Korsmeyer }\end{array}$} \\
\hline & & $\mathrm{r}^{2}$ & $\mathrm{~K}_{0}$ & $\mathrm{r}^{2}$ & $\mathrm{~K}_{1}$ & $\mathrm{r}^{2}$ & $\mathrm{~K}_{\mathrm{h}}$ & $r^{2}$ & $\mathrm{n}$ \\
\hline \multirow{3}{*}{ IBP: PS } & $1: 1$ & 0.79 & 0.85 & 0.89 & 0.006 & 0.97 & 7.58 & 0.98 & 0.31 \\
\hline & $1: 2$ & 0.69 & 0.89 & 0.83 & 0.007 & 0.92 & 8.32 & 0.98 & 0.22 \\
\hline & $1: 3$ & 0.53 & 0.85 & 0.64 & 0.006 & 0.79 & 8.46 & 0.97 & 0.09 \\
\hline \multirow{3}{*}{ IBP: Na CMC } & $1: 1$ & 0.62 & 0.70 & 0.71 & 0.004 & 0.86 & 6.74 & 0.96 & 0.15 \\
\hline & $1: 2$ & 0.62 & 0.76 & 0.73 & 0.005 & 0.86 & 7.22 & 0.89 & 0.15 \\
\hline & $1: 3$ & 0.65 & 0.82 & 0.77 & 0.006 & 0.88 & 7.75 & 0.90 & 0.17 \\
\hline \multirow{3}{*}{ IBP: CCS } & $1: 1$ & 0.73 & 0.74 & 0.82 & 0.004 & 0.94 & 6.74 & 0.95 & 0.27 \\
\hline & $1: 2$ & 0.74 & 0.78 & 0.84 & 0.005 & 0.95 & 7.13 & 0.99 & 0.28 \\
\hline & $1: 3$ & 0.59 & 0.74 & 0.68 & 0.005 & 0.84 & 7.18 & 0.85 & 0.13 \\
\hline \multirow{3}{*}{ IBP: POL407 } & $1: 1$ & 0.74 & 0.75 & 0.84 & 0.005 & 0.94 & 6.92 & 0.97 & 0.27 \\
\hline & $1: 2$ & 0.77 & 0.79 & 0.87 & 0.005 & 0.95 & 7.22 & 0.97 & 0.28 \\
\hline & $1: 3$ & 0.61 & 0.61 & 0.71 & 0.005 & 0.85 & 7.32 & 0.84 & 0.14 \\
\hline Pure IBP & - & 0.90 & 0.39 & 0.92 & 0.002 & 0.96 & 3.31 & 0.83 & 0.45 \\
\hline
\end{tabular}

$\mathrm{KM}=$ Kneading Method, IBP $=$ Ibuprofen, PS $=$ Pregelatinized Starch, NaCMC $=$ Sodium Carboxymethyl cellulose, CCS $=$ Croscarmellose Sodium, POL407 = Poloxamer 407

Fourier Transform infrared spectrophotometer

FTIR spectral studies were carried out for pure drug, physical mixtures (1:3 ratio) and kneading method (1:3 ratio) to check the compatibility between drug and carriers. IR spectra of pure Ibuprofen and SD are shown in figures 7-9. IR spectrum of pure Ibuprofen (Figure 7) was identical with solid dispersion formulations (Figures 8 \& 9). This indicates that there was no interaction between Ibuprofen and carriers in the prepared solid 
dispersions. The spectra of solid dispersions were found to contain identical peaks of pure drug and carrier. In addition, no degradation of drug and carrier due to the high temperature during manufacturing was found from IR spectra.

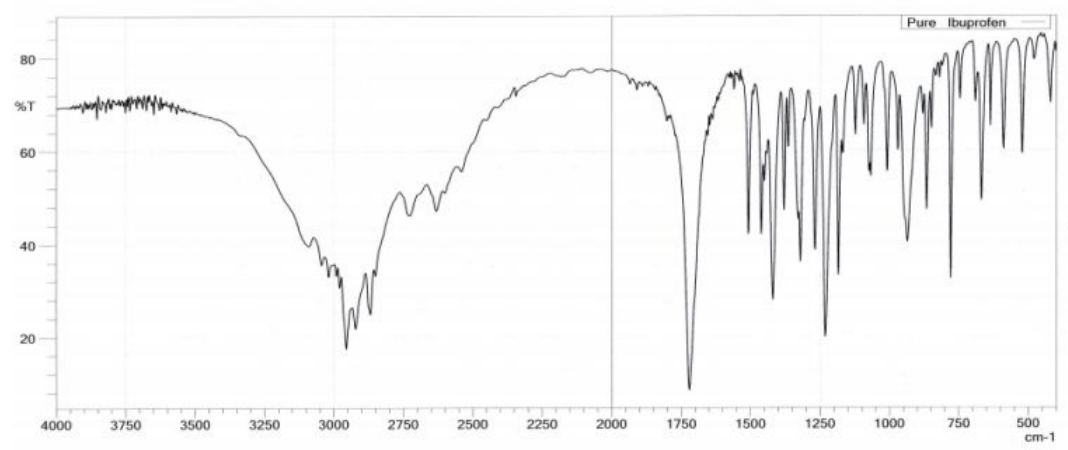

Fig. 7. FTIR spectroscopy of pure Ibuprofen

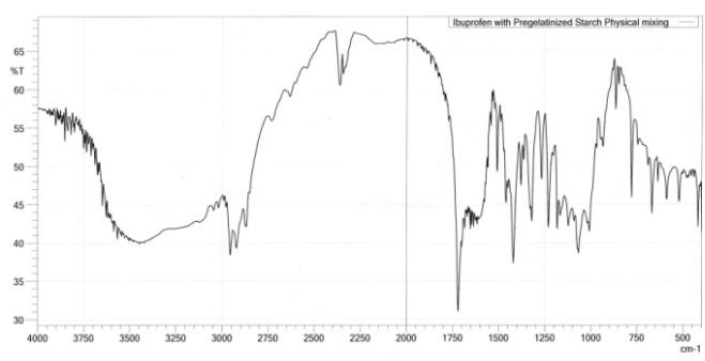

(A)

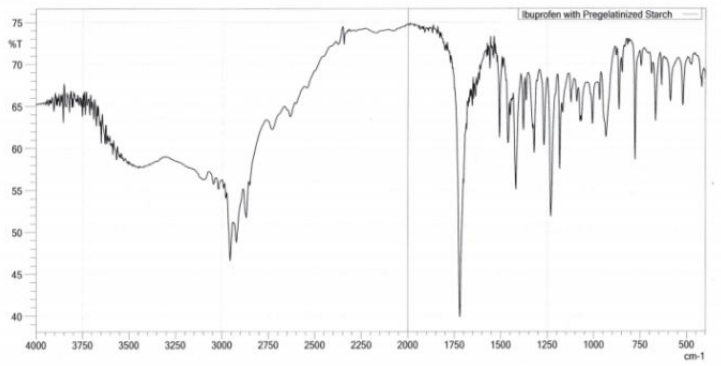

(B)

Fig. 8. FTIR spectroscopy of Ibuprofen and Pregelatinized Starch SD formulation by Physical Mixing (A) and Kneading Methods (B)

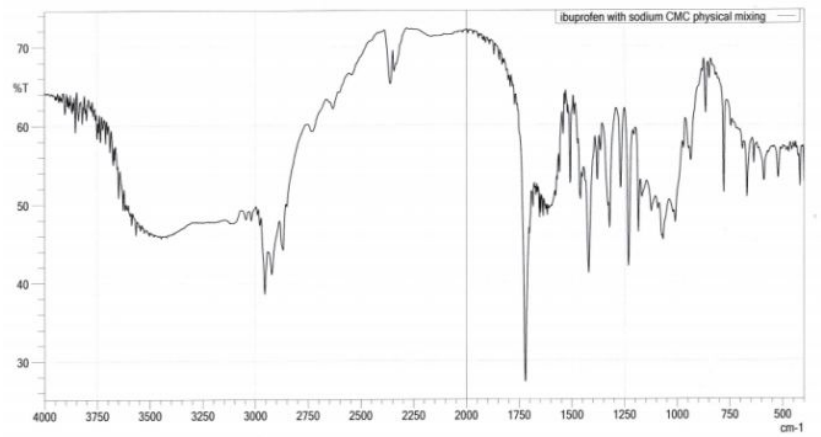

(A)

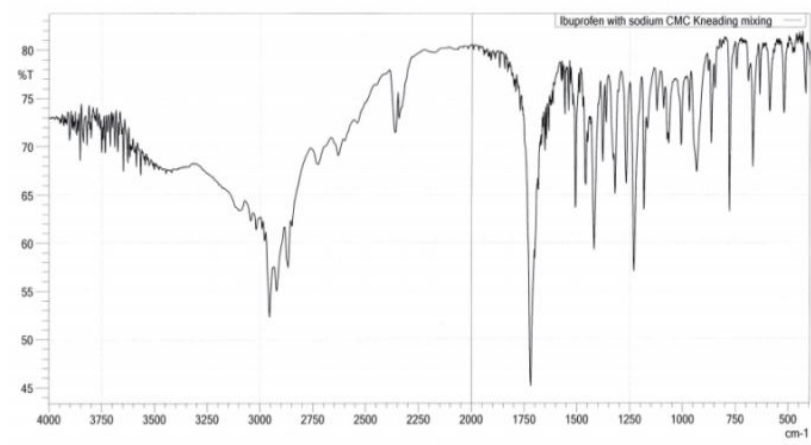

(B)

Fig. 9. FTIR spectroscopy of Ibuprofen and Na-CMC SD formulation by Physical Mixing (A) and Kneading Methods (B)

\section{Conclusion}

It is clear from the discussion that dissolution property of our model drug, Ibuprofen was significantly increased from solid dispersion formulations. Two different techniques were adopted to optimize the Ibuprofen solid dispersion formulations and in case of both techniques, enhanced dissolution was found. Being a BCS class II drug, Ibuprofen entails to be incorporated itself in such formulations which ensures faster dissolution and ultimately more bioavailability. This necessity was completely achieved in our experiment. The characterization through FT-IR analysis also proves that no interaction was happened during formulation development period. 


\section{References}

1. Verheyen, S., N. Blaton, R. Kinget and V. D. Mooter, 2002. Mechanism of Increased Dissolution of Diazepam and Temazepam from Polyethylene Glycol 6000 Solid Dispersions. I. J. Pharm., 249, 45-58.

2. Vanshiv, S. D., M. R. P.Rao, G. S. Sonar, V. K. Gogad and S. G. Borate, 2009. Physicochemical Characterization and In Vitro Dissolution of Domperidone by Solid Dispersion Technique. Indian J. Pharm. Edu. Res., 43, 86-90.

3. Batra,V., V. S. Shirolkar, P. R. Mahaparale, P. V. Kasture and A. D. Deshpande, 2008. Solubility and Dissolution Enhancement of Glipizide by Solid Dispersion Technique. Indian J. Pharm. Edu. Res., 42, 373-378.

4. Serajuddin A., P. C. Sheen and M. A. Agustine. Improved dissolution of a poorly water soluble drug from solid dispersion in polyethylene glycol; polysorbate 80 mixtures. $J$. Pharm. Sci., 1990; 79, 463-464.

5. Amidon, G. L., H. Lennernas, V. P. Shah, and J. R. Crison, 1995. Theoretical basis for a biopharmaceutical drug classification: the correlation of in vitro drug product dissolution and in vivo bioavailability. Pharm Res, 12, 413420 .

6. Serajuddin, A. T. M., 1999. Solid Dispersion of poorly watersoluble drugs Early Promises, Subsequent problems, and recent Breakthroughs. J.of Pharmaceutical Science, 88.

7. Van, E. A., H. A. Van, E. W. Steyerberg, M. Offringa, J. D. Habbema and G. Derksen-Lubsen, 1995. Antipyretic efficacy of ibuprofen and acetaminophen in children with febrile seizures. Archives of Pediatrics \& Adolescent Medicine, 149, 632-710.
8. Tracy, T.S. and S. D. Hall, 1992. Metabolic inversion of (R)ibuprofen: Epimerization and hydrolysis of ibuprofenylcoenzyme A. Drug Metab. Dispos., 20, 322-327.

9. Sharma, A., March 2014-september 2014. London: British Medical Assn. 686-688.

10. Brayfield, A., 2014 (Retrieved 26 June 2014). Ibuprofen.Pharmaceutical Press.

11. Chowdary, K. P. R. and L. Srinivas, 2000. Physical stability and dissolution rate of ibuprofen suspension formulated employing its solid dispersion. Indian J. Pharm. Sci., 62, 253256.

12. Khan, G. M. and Z. Jiabi, 1998. Preparation, characterization and dissolution studies of ibuprofen solid dispersion using polyethylene glycol, talc and PEG-talk as dispersion carriers. Drug Dev. Ind. Pharm., 24, 455- 462.

13. Muralidhar, S., V. Rajesh and V. G., Devalarao. 2010. Fast dissolving celecoxib tablets containing solid dispersion of celecoxib. Int. J. Pharm. Sci. R, 1, 34-40.

14. Korsmeyer, R.W., R. Gurny, E. Doelker, P. Buri, and N. A. Peppas, 1983. Mechanisms of solute release from porous hydrophilic polymers. Int. J. Pharm., 15, 25-35.

15. Peppas, N.A. and J.J., Sahlin, 1989. A simple equation for the description of solute release. III. Coupling of diffusion and relaxation. Int. J. Pharm., 57, 169-172. 